\title{
Circadian activity rhythms for mothers with an infant in ICU
}

\section{Shih-Yu Lee ${ }^{1 *}$, Kathryn A. Lee ${ }^{2}$, Dawn Aycock ${ }^{1}$ and Michael Decker ${ }^{1}$}

1 Byrdine F. Lewis School of Nursing, Georgia State University, Atlanta, GA, USA

2 School of Nursing, University of California at San Francisco, San Francisco, CA, USA

\section{Edited by:}

David Gozal, University of Chicago, USA

\section{Reviewed by:}

Suresh Kotagal, Mayo Clinic College of Medicine, USA

Oliviero Bruni, Sapienza University Rome, Italy

\section{${ }^{*}$ Correspondence:}

Shih-Yu Lee, Byrdine F. Lewis School of Nursing, Georgia State University, P. O. Box 4019, Atlanta, GA, USA. e-mail:slee29@gsu.edu
Circadian rhythms influence sleep and wakefulness. Circadian activity rhythms (CAR) are altered in individuals with dementia or seasonal affective disorder. To date, studies exploring CAR and sleep in postpartum women are rare. The purpose of this report is to describe relationships between CAR, sleep disturbance, and fatigue among 72 first-time mothers during their second week postpartum while their newborn remain hospitalized in intensive care unit. Seventy-two mothers were included in this secondary data analysis sample from three separate studies. Participants completed the general sleep disturbance scale (GSDS), numerical rating scale for fatigue, and a sleep diary. The objective sleep data included total sleep time (TST), wake after sleep onset (WASO), and CAR determined by the circadian quotient (amplitude/mesor) averaged from at least 48-h of wrist actigraphy monitoring. The TST of mothers who self-reported as poor sleepers was $354 \mathrm{~min}$ (SEM =21.9), with a meanWASO of 19.5\% (SEM =2.8). The overall sleep quality measured by the GSDS was clinically, significantly disrupted $(M=5.5, S D=1.2)$. The mean score for morning fatigue was $5.8(S D=2.0)$, indicating moderate fatigue severity. The CAR was 0.62 (SEM = 0.04), indicating poor synchronization. The self-reported good sleepers $(G S D S<3)$ had better CAR ( $M=0.71$, SEM $=0.02)$ than poor sleepers $(G S D S>3)(t[70]=2.0$, $p<0.05)$. A higher circadian equation was associated with higherTST $(r=0.83, p<0.001)$, less WASO $(r=-0.50, p<0.001)$, lower self-reported sleep disturbance scores $(r=-0.35, p=0.01)$, and less morning fatigue $(r=-0.26)$. Findings indicate that mothers with a hospitalized infant have both nocturnal sleep problems and disturbed circadian activity rhythms. Factors responsible for these sleep and rhythm disturbances, the adverse effects on mother's physical and mental well-being, and mother-infant relationship require further study.

Keywords: sleep disturbance, postpartum, ICU, actigraphy, fatigue, amplitude, mesor, circadian activity rhythms

\section{INTRODUCTION}

Maternal stress associated with caring for a newborn requiring intensive medical care initiates a cascade of psychological and physiological sequelae (Miles et al., 1999; Holditch-Davis and Miles, 2000; Lee et al., 2005b). Insomnia is not uncommon as it is one of the first indications of increased anxiety and daily stress (Roehrs et al., 2000). Corollary perturbations in patterns of sleep and wakefulness as well as changes in neurological, endocrine, and immune function are also evident (Lazarus and Folkman, 1984; Lazarus, 1999; Ader, 2000; Opp, 2005).

It is well known that sleep/wake patterns are disrupted in mothers caring for newborns. We hypothesize that circadian activity rhythm (CAR) would also be disturbed for a new mother if her infant remains hospitalized after birth in an intensive care unit (ICU). Circadian processes, which are regulated primarily by the suprachiasmatic nuclei (SCN), are highly influenced by extrinsic stimuli such as ambient illumination and social interactions (Lee et al., 2009). These stimuli may be altered while a newborn infant receives care within the hospital environment. To date, only one report used wrist actigraphy data to address CAR with a very small sample of early stage postpartum women, and better CAR was evident when mothers had more nocturnal total sleep time and less daytime sleep
(Lee and Kimble, 2009). The purpose of this secondary analysis was, therefore, to describe how CAR was related to self-reported sleep disturbances and fatigue severity among mothers with an infant hospitalized in ICU during the early postpartum period (5th- to 12th-day postpartum). We sought to determine (1) what are the sleep and CAR characteristics among mothers with an infant hospitalized in the ICU during their early postpartum period? and (2) what are the relationships between CAR, objective and subjective sleep measures, and maternal fatigue? We hypothesized during the early postpartum stage, mothers characterized as poor CAR will experience more sleep disruption and higher level of fatigue severity compared to women who are good CAR.

\section{MATERIALS AND METHODS}

To further explore the relationships between CAR, sleep, and fatigue, a secondary data analysis was conducted with data from three studies that used the same inclusion/exclusion criteria. The first study (in San Francisco area) was a descriptive correlational study to explore the relationships between maternal sleep and fatigue among 30 Chinese-American women (Lee et al., 2005a). The other two samples (in the Metro Atlanta area) were from studies that tested a sleep intervention to improve sleep and well-being in mothers 
of low-birth weight infants, 20 were from a feasibility study (Lee and Kimble, 2009) and another 22 were from an on-going pilot study. For the last two studies, only the pre-treatment data were analyzed for this paper.

A total of 72 first-time mothers who were in their early postpartum period and with an infant in the ICU were included in this report. Mothers were excluded if any of the following applied: (1) allergic to metal (a contradiction for wrist actigraph), (2) history of an affective illness, (3) current use of medications that may alter sleep (any central nervous system stimulants, depressants, or antidepressant medication), (4) shift worker whose circadian rhythms may already be affected, (5) history of a diagnosed sleep disorder such as sleep apnea or restless legs, which may give a higher waking time from the overnight wrist actigraphy monitoring, (6) under 18 years of age because delayed sleep phase type circadian disorders (late-to-bed, late-to-rise) are very common in adolescents, and (7) mothers who required an extended hospitalization period. A post hoc power analysis was performed; a total sample size of 72 was sufficiently powered to detect a difference in the primary outcome by using an alpha of 0.05 , and at least a medium effect size for $t$-test analysis.

Permission to conduct the study was obtained through the University's Committees on Human Research and through the Institutional Review Boards at hospital recruitment sites. Table 1 summarizes the demographic characteristics of the study participants. Age ranged from 18 to 43 years, and majority of them were Black (40\%) or Asian (41\%). After informed consent, the mothers were asked to wear the wrist actigraph (for objective sleep data) and to press the "event marker button" for their bedtimes and rising

Table 1 | Maternal demographics and characteristics of circadian activity rhythms, sleep, and fatigue $(n=72)$.

\begin{tabular}{|c|c|c|}
\hline Age & Mean & Median \\
\hline & $28.1(\mathrm{SD}=6.6)$ & 28 \\
\hline \multicolumn{3}{|l|}{ ETHNIC GROUP } \\
\hline White & $7(9.7 \%)$ & \\
\hline Black & $29(40.3 \%)$ & \\
\hline Hispanic & $6(8.3 \%)$ & \\
\hline Asian & $30(41.7 \%)$ & \\
\hline \multicolumn{3}{|l|}{ WRIST ACTIGRAPHY DATA } \\
\hline TST & $380(\mathrm{SD}=89)$ & 394 \\
\hline WASO (\%) & $16.8(\mathrm{SD}=11.7)$ & 16.7 \\
\hline \multicolumn{3}{|l|}{ COSINOR ANALYSIS } \\
\hline Amplitude & $69.6(\mathrm{SD}=30)$ & 65.2 \\
\hline Mesor & $104.4(S D=38.6)$ & 115.8 \\
\hline Circadian quotient & $0.68(\mathrm{SD}=0.18)$ & 0.68 \\
\hline Acrophase (time format) & $16: 30$ (range 14:19-20:45) & $16: 15$ \\
\hline Autocorrelation & $0.33(\mathrm{SD}=0.20)$ & 0.35 \\
\hline \multicolumn{3}{|l|}{ GSDS } \\
\hline Total scale & $2.5(\mathrm{SD}=0.9)$ & 2.6 \\
\hline Sleep quality subscale & $3.9(\mathrm{SD}=1.9)$ & 3.7 \\
\hline Sleep quantity subscale & $2.8(\mathrm{SD}=1.2)$ & 3.5 \\
\hline Daytime functioning subscale & $3.1(\mathrm{SD}=1.3)$ & 3.4 \\
\hline \multicolumn{3}{|l|}{ FATIGUE } \\
\hline Morning & $4.8(\mathrm{SD}=2.0)$ & 4.5 \\
\hline
\end{tabular}

times. Mothers were also asked to fill out the following questionnaires and keep a sleep diary to record their bed times and rise times to validate wrist actigraphy data.

The general sleep disturbance scale (GSDS) was used to assess subjective sleep characteristics. The GSDS is a 21 -item, $0-7$ rating scale, which includes seven subscales: sleep onset, waking up during sleep, waking up too early from sleep, quality of sleep, quantity of sleep, daytime functioning, and use of substances to help induce sleep (Lee and DeJoseph, 1992). The GSDS is consistent with the DSM-IV criteria for insomnia symptoms. Higher scores indicate more disturbed sleep, and a mean score of 3 on the total GSDS or any of the subscales represents 3 days per week and is considered a clinically significant sleep disturbance (Lee and DeJoseph, 1992). The Cronbach alpha coefficient for the overall scale was 0.80 in the current sample.

The Lee fatigue scale (LFS) is 13-item numerical rating scale with high internal consistency reliability in previous research (Lee et al., 1991). It measures fatigue severity in the morning (rising time) using numerical rating scales from 0 (not at all) to 10 (extremely), and morning score of 3.3 or higher indicates clinical significant fatigue. To reduce participant burden and redundancy of items, only 7 of the 13 fatigue items were used and the Cronbach alpha coefficients for the seven-item version remained high, ranging from 0.86 to 0.96 in this sample.

Wrist actigraph was used to estimate nocturnal total sleep time (TST) and wake after sleep onset (WASO) for 48-72 h (Mini Motionlogger Actigraphy, octagonal motionlogger, Ambulatory Monitoring Inc., Ardsley, NY, USA). The wrist actigraph also measures activity level during the day, and the continuous 24-h data are used to estimate CAR (Ancoli-Israel et al., 2003). The zerocrossing mode automatic sleep-scoring program (Action 4 Software Program, Ambulatory Monitoring Inc., Ardsley, NY, USA) was used to calculate TST and WASO. The mesor (24-h adjusted mean), the amplitude (mesor to peak), the acrophase (peak time of activity), and the autocorrelation for a 24-h rhythm were also determined using Action 4 software. Circadian quotient (amplitude/mesor) was used to indicate CAR (Ancoli-Israel et al., 1997). Mothers were dichotomized at the median circadian quotient for good and poor CAR groups. Descriptive statistics were used to characterize the sample and describe study variables. Interval/ratio level data were examined and noted to meet the assumptions for the selected statistical methods. Pearson correlations were used to examine relationships between objective and subjective measures of sleep, acrophase, amplitude, mesor, circadian quotient, and fatigue severity. $t$-Tests were used to test for differences in self-reported sleep and fatigue severity between good CAR and poor CAR mothers.

\section{RESULTS}

Mothers self-reported a clinically significant poor sleep quality $(3.9 \pm 1.9)$ and disrupted daytime functioning $(3.1 \pm 1.3)$ from the GSDS subscale. Objective sleep data from wrist actigraphy indicated about $6.3 \mathrm{~h}$ of nocturnal TST and a WASO average of $16.8 \%$, indicating these mothers experienced disrupted sleep. Cosinor analysis indicated the amplitude was $69.6(\mathrm{SD}=29.7)$, the mesor was 104.4 $(\mathrm{SD}=38.6)$, and the circadian quotient ranged from 0.19 to 1.02 , with a mean of $0.68(\mathrm{SD}=0.18)$ and a median of 0.68 . The autocorrelation for the fit to a $24-\mathrm{h}$ rhythm was $0.33(\mathrm{SD}=0.20)$. The acrophase in 
military time format ranged from 14:19 to 20:45 (median 16:15), with a later acrophase indicates of a more delayed circadian phase (AncoliIsrael et al., 1997). Mothers also reported clinically significant fatigue upon awakening in the morning (mean $=4.8, \mathrm{SD}=2.0$ ), indicating that they did not feel refreshed from their night's sleep.

The relationships between CAR, sleep, and fatigue are detailed in Table 2. Mothers who had a higher circadian quotient (better CAR) had more nocturnal TST $(r=0.83, p<0.01)$, less WASO $(r=-0.50, p<0.01)$, and less self-reported disturbed sleep from the GSDS $(r=-0.35, p<0.05)$. Amplitude was statistically significantly correlated with TST $(r=0.49, p<0.01)$, morning fatigue $(r=-0.37$, $p<0.01)$, and disturbed sleep quality $(r=-0.30, p<0.01)$, indicating those who have robust rhythms had a longer nocturnal sleep, less fatigue severity, and perceived better sleep quality (Table 4). The younger the mother, the higher their amplitude $(r=-0.43$, $p<0.01)$ and mesor $(r=-0.42, p<0.01)$. Acrophase was associated with WASO $(r=0.44, p<0.01)$, GSDS $(r=0.26, p<0.05)$, and poor daytime functioning $(r=0.43, p<0.01)$, indicating those mothers with a later time of peak activity had more night-time awakenings and experienced poor daytime functioning.

To date, little is known about CAR for postpartum women. Thus, circadian quotient was dichotomized at the median (0.68) to further explore sleep and fatigue characteristics among these 72 mothers with poor CAR (circadian quotient $<0.68$ ) and good CAR (circadian quotient $\geq 0.68$ ). The characteristics of sleep, fatigue, and CAR for both groups are listing in Table 3. Mothers in the poor CAR group had a statistically significant lower autocorrelation $(t[70]=3.52, p=0.001)$, less nocturnal sleep time $(t[70]=7.16, p<0.001)$, more self-reported sleep disturbance $(t[70]=2.30, p<0.03)$, and poorer self-reported sleep quality $(t[70]=2.64, p=0.01)$ compared to the high CAR mothers. To further explore subjective sleep characteristics, mothers were categorized into good sleepers (GSDS mean score $<3$ ) and poor sleepers (GSDS mean score $<3$ ). Table 4 shows characteristics of sleep, fatigue, and CAR in good sleepers and poor sleepers.

\section{DISCUSSION}

Research on sleep for early postpartum stage women with a hospitalized infant, either pre-term or full-term with a medical problem, is very limited. The results from this study supported our hypothesis that mothers with poor CAR experienced more sleep disruption and higher fatigue severity compared to those with good CAR. These early stage postpartum women's sleep quality and quantity were considerably poor, their CAR is not well synchronized, and they also experienced clinically significant morning fatigue severity. The nocturnal TST of $6.3 \mathrm{~h}$ from the study participants is far from the recommended 7-8 h (NSF, 2005).

Table 2 | Correlations between circadian activity rhythms, sleep, and fatigue $(n=72)$.

\begin{tabular}{|c|c|c|c|c|c|}
\hline & Amplitude & Mesor & Circadian quotient & Acrophase & Autocorrelation \\
\hline TST & $0.43^{* *}$ & -0.17 & $0.83^{* *}$ & -0.10 & $0.47^{* *}$ \\
\hline WASO & -0.02 & $0.32 * *$ & $-0.50 * *$ & $0.44^{* *}$ & $-0.32 * *$ \\
\hline GSDS & -0.15 & -0.04 & $-0.35^{*}$ & $0.26^{*}$ & $-0.24^{*}$ \\
\hline Sleep quality & $-0.30 * *$ & $-0.24^{*}$ & -0.23 & -0.10 & -0.01 \\
\hline Sleep quantity & 0.02 & 0.09 & $-0.34^{*}$ & 0.23 & -0.23 \\
\hline Daytime dysfunction & 0.04 & 0.12 & -0.22 & $0.43^{* *}$ & $-0.24^{*}$ \\
\hline Morning fatigue & $-0.37^{* *}$ & $-0.26^{* *}$ & -0.26 & 0.11 & -0.18 \\
\hline Age & $-0.43^{* *}$ & $-0.42^{* *}$ & -0.20 & -0.10 & -0.18 \\
\hline
\end{tabular}

GSDS, general sleep disturbance; TST, total sleep time monitored from wrist actigraph; WASO, wake after sleep onset. ${ }^{*} p<0.05 .{ }^{*} p<0.01$.

Table 3 | Sleep and fatigue characteristics based on circadian quotient.

\begin{tabular}{lll}
\hline & $\begin{array}{l}\text { Good circadian activity rhythms } \\
\text { circadian quotient } \mathbf{0 . 6 8}(\boldsymbol{n}=\mathbf{3 6})\end{array}$ & $\begin{array}{l}\text { Poor circadian activity rhythms circadian } \\
\text { quotient }<\mathbf{0 . 6 8}(\boldsymbol{n}=\mathbf{3 6})\end{array}$ \\
\hline Cosinor analysis Mesor & $101.6(\mathrm{SEM}=5.8)$ & $107.2(\mathrm{SEM}=7.1)^{* *}$ \\
Amplitude & $83.0(\mathrm{SEM}=4.7)$ & $56.2(\mathrm{SEM}=4.1)^{* *}$ \\
Autocorrelation & $0.41(\mathrm{SEM}=0.03)$ & $0.25(\mathrm{SEM}=0.03)^{* *}$ \\
Acrophase & $16: 28$ & $16: 33$ \\
TST & $437(\mathrm{SEM}=10)$ & $323(\mathrm{SEM}=12)^{* *}$ \\
WASO & $14.7(\mathrm{SEM}=1.4)$ & $18.8(\mathrm{SEM}=2.3)$ \\
GSDS & $2.3(\mathrm{SD}=0.9)$ & $2.7(\mathrm{SD}=0.8)^{* *}$ \\
Sleep quality & $3.3(\mathrm{SD}=2.0)$ & $4.5(\mathrm{SD}=1.8)^{* *}$ \\
Sleep quantity & $2.4(\mathrm{SD}=1.4)$ & $3.1(\mathrm{SD}=0.8)^{* *}$ \\
Daytime function & $2.9(\mathrm{SD}=1.2)$ & $3.2(\mathrm{SD}=1.4)$ \\
Morning fatigue & $4.4(\mathrm{SEM}=0.3)$ & $5.2(\mathrm{SEM}=0.3)$ \\
\hline
\end{tabular}

GSDS, general sleep disturbance; TST, total sleep time monitored from wrist actigraph; WASO, wake after sleep onset. ${ }^{*} p<0.05 .{ }^{*} p<0.01$. 
Table 4 | Circadian activity rhythm, sleep, and fatigue characteristics based on GSDS.

\begin{tabular}{|c|c|c|}
\hline & $\begin{array}{l}\text { Good sleepers } \\
\text { GSDS less than } 3 \\
(n=45)\end{array}$ & $\begin{array}{l}\text { Poor sleepers } \\
\text { GSDS equal or } \\
\text { above } 3 \text { ( } n=27 \text { ) }\end{array}$ \\
\hline Cosinor analysis Mesor & $104($ SEM = 5.9) & $105($ SEM $=7.3)$ \\
\hline Amplitude & $72.6(\mathrm{SEM}=4.2)$ & $64.6(\mathrm{SEM}=6.3)$ \\
\hline $\begin{array}{l}\text { Acrophase (military } \\
\text { time format) }\end{array}$ & $16: 20$ & $16: 48$ \\
\hline Autocorrelation & $0.35($ SEM = 0.03) & $0.3(\mathrm{SEM}=0.04)$ \\
\hline Circadian quotient & $0.71(\mathrm{SEM}=0.02)$ & $0.62(\mathrm{SEM}=0.04)^{*}$ \\
\hline TST & 395 (SEM = 9.6) & $353(\text { SEM }=22)^{*}$ \\
\hline WASO & $15($ SEM = 1.7) & $19.5(\mathrm{SEM}=2.8)$ \\
\hline GSDS & $1.9(\mathrm{SD}=0.6)$ & $3.4(\mathrm{SD}=0.3)^{* *}$ \\
\hline Sleep quality & $3.3(\mathrm{SD}=1.9)$ & $5.4(\mathrm{SD}=1.2)^{* *}$ \\
\hline Sleep quantity & $2.4(S D=1.4)$ & $3.4(S D=0.4)^{* *}$ \\
\hline Daytime sleepiness & $2.4(S D=1.3)$ & $4.0(\mathrm{SD}=0.6)^{* *}$ \\
\hline Morning fatigue & $4.2(\mathrm{SEM}=0.3)$ & $5.8(\text { SEM }=0.4)^{* *}$ \\
\hline
\end{tabular}

GSDS, general sleep disturbance; TST, total sleep time monitored from wrist actigraph; WASO, wake after sleep onset. ${ }^{*} p<0.05$. ${ }^{*} p<0.01$.

Nocturnal TST for first-time mothers with a healthy infant has been documented as less than $7 \mathrm{~h}$ in the first month of the postpartum period (Gay et al., 2004). It is understandable that mothers with a healthy newborn at home are likely to be deprived of sleep because their newborns demand care; however, mothers in this study reported similar nocturnal TST even though their infants were still in the hospital. Since sleep loss increases risk of cardiovascular disorders and type 2 diabetes (Alvarez and Ayas, 2004), it is important to monitoring these physical problems for this population of women if they suffered long-term sleep deprivation.

Data regarding CAR is very rare and there is almost no existing record for postpartum women. A landmark study used wrist actigraphy to monitor 23 healthy adults ( 10 males and 13 females), aged 22-54 years, for 5 days; the mesor, amplitude, and circadian quotient for the female participants was 151.7, 115.8, and 0.76, respectively, and the acrophase was between 13:42 and 15:18 (Brown et al., 1990). A recent study investigating CAR among breast cancer patients found that their mesor and amplitude prior to chemotherapy were $132.3(\mathrm{SD}=24.6)$ and $97.2(\mathrm{SD}=22.8)$. The mean circadian quotient was 0.73 and a more robust activity rhythm was associated with lower fatigue and higher performance status for women during chemotherapy for breast cancer (Berger et al., 2009). Yet, the postpartum mothers in these three samples experienced lower amplitude, mesor, and circadian quotient compared to other populations reported in the literature. Differences for our study participants may be a result of their early postpartum stage, distress due to their unexpected early childbirth, and their infant's medical condition.

Circadian activity rhythm has also been found to be related to the severity of dementia (Ancoli-Israel et al., 1997; Gehrman et al., 2005) and seasonal affective disorder (Winkler et al., 2005). The cosinor analyses indicated that mothers in this study experienced poor CAR; the associations between CAR and sleep and fatigue suggest an individual with a better CAR will perceive better sleep and less fatigue severity. Poor CAR could have resulted from multiple factors, such as stressful life events due to their infant's medical conditions, postpartum hormonal issues, and poor sleep hygiene, including light exposure levels (Lee, 2003). In general, the neonatal ICU is surrounded by artificial dim light to minimize the stimulus for infants and promote their development. In addition to their distressed emotional status, the ICU environment has persistent exposure to lighting and, coupled with the loss of a discrete diurnal light/dark cycle within the hospital, maternal sleep disruption may be exacerbated. All of these factors need to be further explored to better understand altered CAR and sleep patterns for this specific subgroup of postpartum women. Prolonged exposure to artificial light may alter the entrainment of the SCN and circadian pacemaker, and we would speculate that loss of entrainment to light/dark cycles leads to self-reported sleep problems and excessive fatigue.

The American Academy of Sleep Medicine recommends wrist actigraphy monitoring for at least $72 \mathrm{~h}$; however, the minimum needed to obtain sufficient peaks and troughs is $36 \mathrm{~h}$ to calculate 24-h rhythm parameters. The majority of the study participants only had 48-h activity data due to subject burden. An extended activity monitoring should increase the internal validity for the findings. However, in this study, similar characteristics were found between the good and poor sleepers classified by using the GSDS and by using the circadian quotient median split. Mothers who had a higher self-reported more general sleep disturbances, had a poor circadian quotient, and more sever morning fatigue. Mothers with poor circadian quotient had higher self-reported sleep disturbances and had more severe morning fatigue. In addition, there was a statistically significant association between poor circadian quotient and poor self-report general sleep disturbances. From the correlations between the objective and subjective measurements, it can be said that if an individual self-reported sleep disruption, we should also evaluate their activity rhythms. Since wearing a wrist actigraph may become a burden to participants, and an actigraph is not generally available in most clinical settings, clinician can use GSDS for an initial assessment in this population of mother.

Although this study has added some new knowledge, findings from this present study need to be interpreted with caution due to some limitations. For example: (1) lack of a control group of mothers whose babies were being cared for at home, (2) monitoring of how long the mothers spent at the NICU under ambient illumination was absent, (3) some confounding variables such as the levels of maternal stress and anxiety could be different based on their infant gestational age and their other life events, and (4) contextual variables such as ethnicity could not be controlled in the study. Thus, the internal validity and generalizability may be limited.

Researchers have pointed out that promoting sleep during the early postpartum period is an important part of improving childbearing women's quality of life (Huang et al., 2004). Postpartum fatigue has been described as a pathological state which persists through the circadian rhythm and cannot be relieved through a single period of sleep (Milligan et al., 1996). Our findings document short nocturnal sleep time in the early postpartum period, and that self-reported poor sleepers have significantly less nocturnal TST, more disturbed daytime functioning, higher fatigue severity, and 
more poorly synchronized CAR compared to the good sleepers. Both good and poor sleepers in these three samples experienced disrupted sleep (short TST and high WASO), poor sleep quality, as well as clinically significant fatigue. Health care providers should assess maternal sleep quality and quantity, particularly during the early stage of the postpartum period. Sleep is a fundamental human need and provides physical and psychological restoration. Relationships between CAR and self-reported sleep disturbance and fatigue severity were evident. Poor sleep and CAR promote a high tendency for diminished cognitive ability, decision-making,

\section{REFERENCES}

Ader, R. (2000). On the development of psychoneuroimmunology. Eur. J. Pharmacol. 405, 167-176.

Alvarez, G. G., and Ayas, N. T. (2004). The impact of daily sleep duration on health: a review of the literature. Prog. Cardiovasc. Nurs. 19, 56-59.

Ancoli-Israel, S., Clopton, P., Klauber, M. R., Fell, R., and Mason, W. (1997). Use of wrist activity for monitoring sleep/ wake in demented nursing-home patients. Sleep 20, 24-27.

Ancoli-Israel, S., Cole, R., Alessi, C., Chambers, M., Moorcroft, W., and Pollak, C. P. (2003). The role of actigraphy in the study of sleep and circadian rhythms. Sleep 26, 342-392.

Berger, A. M., Wielgus, K., Hertzog, M., Fischer, P., and Farr, L. (2009). Patterns of circadian activity rhythms and their relationships with fatigue and anxiety/ depression in women treated with breast cancer adjuvant chemotherapy. Support Care Cancer 18, 105-114.

Brown, A.C., Smolensky, M.H., D'Alonzo, G. E., and Redman, D. P. (1990). Actigraphy: a means of assessing circadian patterns in human activity. Chronobiol. Int. 7, 125-133.

Gay, C. L., Lee, K. A., and Lee, S. Y. (2004). Sleep patterns and fatigue in new mothers and fathers. Biol. Res. Nurs. 5, 311-318.

Gehrman, P., Marler, M., Martin, J. L., Shochat, T., Corey-Bloom, J., and Ancoli-Israel, S. (2005). The relationship between dementia severity and rest/activity circadian rhythms. Neuropsychiatr. Dis. Treat. 1, 155-163.
Holditch-Davis, D., and Miles, M. S. (2000). Mothers' stories about their experiences in the neonatal intensive care unit. Neonatal Netw. 19, 13-21.

Huang, C. M., Carter, P. A., and Guo, J. L. (2004). A comparison of sleep and daytime sleepiness in depressed and non-depressed mothers during the early postpartum period. J. Nurs. Res. 12, 287-296.

Lazarus, R. S. (1999). Stress and Emotion: A New Synthesis. New York: Springer Publishing Company.

Lazarus, R. S., and Folkman, S. (1984). Stress, Apraised, and Coping. New York: Springer.

Lee, K. A. (2003). “Impaired sleep,” in Pathophysiological Phenomena in Nursing, 3rd Edn., eds V. CarrieriKohlman, A. M. Lindsey, and C. M. West (St. Louis: Saunders), 363-385.

Lee, K. A., and DeJoseph, J. F. (1992). Sleep disturbances, vitality, and fatigue among a select group of employed childbearing women. Birth 19, 208-213.

Lee, K. A., Hicks, G., and Nino-Murcia, G. (1991). Validity and reliability of a scale to assess fatigue. Psychiatry Res. 36, 291-298.

Lee, M.L., Swanson, B. E., and de la Iglesia, H. O. (2009). Circadian timing of REM sleep is coupled to an oscillator within the dorsomedial suprachiasmatic nucleus. Curr. Biol. 19, 848-852.

Lee, S. Y., and Kimble, L. P. (2009). Impaired sleep and well-being in mothers with low-birth-weight infants. J. Obstet. Gynecol. Neonatal Nurs. 38, 676-685.

and performance (Leff et al., 2010; Steinborn et al., 2010); thus, the potentially adverse impacts to safe infant care, maternal learning, and role competency need to be further explored.

\section{ACKNOWLEDGMENTS}

Shih-Yu Lee was supported by T32NR 07088, the Graduate Diversion at University of California, San Francisco, AWHONN, the intramural grant at Georgia State University, and the NIH/ NINR (1R15NR010152-01A1). The authors thank the volunteers who participated in the studies for making it possible.

Lee, S. Y., Lee, K. A., Rankin, S. H. Alkon, A., and Weiss, S. J. (2005a) Acculturation and stress in ChineseAmerican parents of infants cared for in the ICU. Adv. Neonatal Care 5 , 315-325.

Lee, S. Y., Lee, K. A., Rankin, S. H., Weiss, S., and Alkon, A. (2005b). ICU stress and sleep disturbance among ChineseAmerican parents. Sleep 28, A344.

Leff,D.R.,Orihuela-Espina, F.,Athanasiou, T., Karimyan, V., Elwell, C., Wong, J. Yang, G. Z., and Darzi, A. W. (2010) "Circadian cortical compensation": a longitudinal study of brain function during technical and cognitive skills in acutely sleep-deprived surgical residents. Ann. Surg. 252, 1082-1090.

Miles, M. S., Wilson, S. M., and Docherty, S. L. (1999). African American mothers' responses to hospitalization of an infant with serious health problems. Neonatal Netw. 18, 17-25.

Milligan, R., Lenz, E. R., Parks, P. L., Pugh, L. C., and Kitzman, H. (1996). Postpartum fatigue: clarifying a concept. Sch. Inq. Nurs. Pract. 10 279-291.

NSF. (2005). Helping yourself to a good night's sleep. Retrieved October 2 , 2005, from www.sleepfoundation. org

Opp, M. R. (2005). Cytokines and sleep. Sleep Med. Rev. 9, 355-364.

Roehrs, T., Zorick, F. J., and Roth, T. (2000). "Transient and short-time insomnias," in Principles and practice of sleep medicine, 3rd Edn. eds M. H. Kryger, T. Roth, and W. C. Dement (Philadelphia: Saunders), 624-632.
Steinborn, M. B., Bratzke, D., Rolke, B., Gordijn, M. C., Beersma, D. G., and Ulrich, R. (2010). The effect of 40 hours of constant wakefulness on number comparison performance. Chronobiol. Int. 27, 807-825.

Winkler, D., Pjrek, E., Praschak-Rieder, N., Willeit, M., Pezawas, L., Konstantinidis, A., Stastny, J., and Kasper, S. (2005). Actigraphy in patients with seasonal affective disorder and healthy control subjects treated with light therapy. Biol. Psychiatry 58, 331-336.

Conflict of Interest Statement: The authors declare that the research was conducted in the absence of any commercial or financial relationships that could be construed as a potential conflict of interest.

Received: 31 October 2010; accepted: 08 December 2010; published online: 23 December 2010.

Citation: Lee S-Y, Lee KA, Aycock D and Decker M (2010) Circadian activity rhythms for mothers with an infant in ICU. Front. Neur. 1:155. doi: 10.3389/ fneur.2010.00155

This article was submitted to Frontiers in Sleep and Chronobiology, a specialty of Frontiers in Neurology.

Copyright (c) 2010 Lee, Lee, Aycock and Decker. This is an open-access article subject to an exclusive license agreement between the authors and the Frontiers Research Foundation, which permits unrestricted use, distribution, and reproduction in any medium, provided the original authors and source are credited. 